\title{
Communication in XYZ All-to-All Quantum Networks with a Missing Link
}

\author{
Sougato Bose \\ Department of Physics and Astronomy, University College London, London WC1E 6BT, United Kingdom. \\ Andrea Casaccino \\ Computer Architecture Group Lab, Information Engineering Department, University of Siena, 53100 Siena, Italy \\ Stefano Mancini \\ Department of Physics, University of Camerino, I-62032 Camerino, Italy \\ Simone Severini \\ Institute for Quantum Computing and Department of Combinatorics 83 Optimization, \\ University of Waterloo, Waterloo N2L 3G1, ON Canada
}

(Dated: October 11, 2018)

\begin{abstract}
We explicitate the relation between Hamiltonians for networks of interacting qubits in the $X Y Z$ model and graph Laplacians. We then study evolution in networks in which all sites can communicate with each other. These are modeled by the complete graph $K_{n}$ and called all-to-all networks. It turns out that $K_{n}$ does not exhibit perfect state transfer (PST). However, we prove that deleting an edge in $K_{n}$ allows PST between the two non-adjacent sites, when $n$ is a multiple of four. An application is routing a qubit over $n$ different sites, by switching off the link between the sites that we wish to put in communication. Additionally, we observe that, in certain cases, the unitary inducing evolution in $K_{n}$ is equivalent to the Grover operator.
\end{abstract}

\section{INTRODUCTION}

Networks of interacting qubits are a generalization of spin chains. These are of theoretical importance for the study of many-body quantum systems and could constitute a good test ground for technologies spanning from quantum key distribution in multi-user networks to various nano-scale devices.

Since the first works [2, 5, 10] (see also [3], for a review), networks of interacting qubits are considered to be good candidates for engineering perfect quantum channels and allow information transfer between distant particles. In this perspective, such networks appear to be useful for the implementation of data buses in quantum mechanical devices, in particular because undergoing a free dynamics after an initial set-up. One of the problems arising in the scenario is given by natural dispersion effects, which determine a loss of information often proportional to the distance (i.e., the number of spins) between communicating sites. Ways to circumvent the issue are based on a local tuning of the couplings [5] or protocols for lifting the encoding of qubits into multiparticle states [4, 9] (see also [6] and the references therein).

Another approach is to study what network topologies guarantee a small decay of fidelity, when the couplings are homogeneous and constant during the entire evolution of the system. Results in this direction have provided examples of the so-called perfect state transfer (for short, $P S T$ ), in relation to combinatorial properties of the graphs modeling the networks (a list of references on this area is in [1]). In the $X Y$ model, when considering a single excitation, it has been shown that PST depends essentially on the eigensystem of the adjacency matrix of the graph, because certain invariant eigenspaces of the total Hilbert space evolve independently. In the cases analyzed so far, even if PST occurs between two specific sites of a network, routing arbitrarily over the entire network still requires an external controller.

Here we will deal with PST in certain networks of spin-half particles with the $X Y Z$ interaction. Specifically, we will describe the structure of the Hamiltonian which governs the single excitation setting. This turns out to be proportional to the (combinatorial) Laplacian of the graph modeling the network. Thus, it may be worth to remark that Hamiltonian arising in the $X Y$ and the $X Y Z$ interactions are naturally associated to the adjacency matrix and the Laplacian respectively and that these objects are the two most common matrix representations of graphs.

We will study quantum evolution in a network in which all sites can communicate between each other (in both directions). Such a network is modeled by the complete graph on $n$ vertices $K_{n}$ and it is called all-to-all network. The number of links in $K_{n}$ is $n(n-1) / 2$. It turns out that $K_{n}$ does not exhibit PST. However, we will show that deleting an edge in $K_{n}$ will allow PST between the two non-adjacent sites in the obtained network, when $n$ is a multiple of 4 (apart the trivial case $n=2$ ). This is modeled by a graph usually denoted by $K_{n}^{-}$, an all-toall network with a missing link. The phenomenon is due to interference effects and it is counterintuitive. On the side, we remark that in certain cases the unitary inducing evolution in $K_{n}$ is the Grover matrix up to an overall phase.

An application is routing a qubit on $K_{n}^{-}$(i.e., transferring the qubit between any two vertices of $K_{n}^{-}$, arbitrarily). The protocol involves an external agent, whose role is to switch OFF only the link between the sites that 
we wish to put in communication. When the process involves all $n$ sites, it requires exactly $2 n-1 \mathrm{ON} / \mathrm{OFF}$ switching operations. This does not provide any direct advantage over considering the empty graph as a network (this is trivially the graph with no edges), with a single edge $i j$ if communication between site $i$ and site $j$ is desired. On the other hand, dynamics on $K_{n}^{-}$is far more complex and the same edge configuration is potentially useful for other tasks beyond perfect communication of a qubit.

The structure of the paper is as follows. In Section 2 we give minimal background information on the $X Y Z$ model and the necessary mathematical definitions. We will clarify the exact relation between the $X Y Z$ Hamiltonian and the Laplacian matrix (this relation was mentioned in [7], but not given explicitly). In Section 3 we study evolution on $K_{n}$. Section 4 is devoted to $K_{n}^{-}$. Wi will also mention a generalization in which we delete an arbitrary number of vertex disjoint edges. A brief conclusion is drawn in Section 5 .

\section{SET-UP}

Let $G=(V, E)$ be a simple undirected graph (that is, without loops or parallel edges), with set of vertices $V(G)$ and set of edges $E(G)$. We take $V(G)=\{1, \ldots, n\}$ and assume that $|E(G)|=m$. The degree $d(i)$ of a vertex $i$ is the number of edges incident with $i$. The adjacency matrix of $G$ is denoted by $A(G)$ and defined by $[A(G)]_{i j}=1$, if $i j \in E(G) ; 0,[A(G)]_{i j}=0$ if $i j \notin E(G)$.

The adjacency matrix is a useful tool to describe a network of $n$ spin-half quantum particles. The particles are usually attached to the vertices of $G$, while the edges of $G$ represent their allowed couplings. If one considers the $X Y Z$ interaction model (isotropic Heisenberg model), then $\{i, j\} \in E(G)$ means that the particles $i$ and $j$ interact by the Hamiltonian $[H(G)]_{i j}=$ $\left(X_{i} X_{j}+Y_{i} Y_{j}+Z_{i} Z_{j}\right)$, where $X_{i}, Y_{i}$ and $Z_{i}$ are the Pauli operators of the $i$-th particle (here we consider unit coupling constant). In extension of the $X Y$ model, we decide to call this the $X Y Z$ model, as the $Z$ interaction has been added to the $X Y$ Hamiltonian. Thus, the Hamiltonian of the whole network reads

$$
H_{X Y Z}(G)=\frac{1}{2} \sum_{i \neq j}[A(G)]_{i j}\left(X_{i} X_{j}+Y_{i} Y_{j}+Z_{i} Z_{j}\right),
$$

and it acts on the Hilbert space $\mathcal{K}=\left(\mathbb{C}^{2}\right)^{\otimes n}$.

Let us now restrict our attention to the single excitation subspace $\mathcal{H} \cong \mathbb{C}^{n}$, i.e., the subspace of dimension $n$ spanned by the vectors $\{|1\rangle, \ldots,|n\rangle\}$. A vector $|j\rangle$ indicates the presence of the excitation on the $j$-th site and the absence on all the others. This is equivalent to the following tensor product of the $Z$ eigenstates $|\underbrace{0 \ldots 010 \ldots 0}_{n}\rangle$, being 1 in the $j$-th position. In the basis $\{|1\rangle, \ldots,|n\rangle\}$, the Hamiltonian coming from Eq. (1) has the following entries

$$
\left[H_{X Y}(G)\right]_{i j}=2[A(G)]_{i j}, \quad i \neq j
$$

and

$$
\left[H_{Z}(G)\right]_{i i}=\frac{1}{2} \sum_{i, j}[A(G)]_{i j}-2 \sum_{j}[A(G)]_{i j}
$$

Eq. (2) comes from the term $X_{i} X_{j}+Y_{i} Y_{j}$ and Eq. (3) comes from the term $Z_{i} Z_{j}$. It is then clear that in the $X Y$ model the relation between the Hamiltonian and adjacency matrix simply reduces to $\left[H_{X Y}(G)\right]_{i j}=2[A(G)]_{i j}$. Here, $H_{X Y Z}(G)=H_{X Y}(G)+H_{Z}(G)$. By associating the vertex $i \in V(G)$ to the vector $|i\rangle \in\{|1\rangle, \ldots,|n\rangle\}$, we can introduce the following modified version of the adjacency matrix:

Definition 1 The XYZ adjacency matrix of a graph $G$ is denoted by $H(G)$ and defined by

$$
\left[H_{X Y Z}(G)\right]_{i j}= \begin{cases}2, & \text { if } i j \in E(G) ; \\ 0, & \text { if } i j \notin E(G) ; \\ m-2 d(i), & \text { if } i=j .\end{cases}
$$

We shall drop the subscript $X Y Z$ because in this paper we only deal the $X Y Z$ model. The matrix $H(G)$ has a neat relation to the Laplacian of $G$. Let $\Delta(G)$ be an $n \times n$ diagonal matrix such that $[\Delta(G)]_{i i}=d(i)$. The (combinatorial) Laplacian of $G$ is the matrix $L(G):=$ $\Delta(G)-A(G)$. Let $I_{n}$ be the $n \times n$ identity matrix.

Lemma 2 The $X Y Z$ adjacency matrix of a graph $G$ is $H(G)=m I_{n}-2 L(G)$.

Proof. By Definition 1 $H(G)=2 A(G)+m I_{n}-2 \Delta(G)$. By combining this fact with the definition of Laplacian, we obtain the desired expression.

Corollary 3 Given any graph $G$, the matrices $L(G)$ and $H(G)$ have a common set of eigenvectors. Moreover, if $\mu$ is an eigenvalue of $L(G)$ then $\lambda=m-2 \mu$ is an eigenvalue of $H(G)$.

Let $\{|1\rangle, \ldots,|n\rangle\}$ be the standard basis of an Hilbert space $\mathcal{H} \cong \mathbb{C}^{n}$. We associate the vertex $i \in V(G)$ to the vector $|i\rangle \in\{|1\rangle, \ldots,|n\rangle\}$. Let $\iota=\sqrt{-1}$. Given a graph $G$, two of its vertices $i$ and $j$, and a real number $0<t<\infty$, the fidelity at time $t$ between vertex $i$ and vertex $j$ is defined by $f_{G}(i, j, t):=\left|\left\langle j\left|e^{-\iota H(G) t}\right| i\right\rangle\right|$. We say that two vertices $i$ and $j$ of a graph $G$ admits perfect state transfer (w.r.t. the XYZ adjacency matrix) if there exists $t$ for which $f_{G}(i, j, t)=1$. Let $U_{t}(G)=e^{-\iota H(G) t}$ be the unitary matrix associated to $H(G)$ as a function of $t$. Therefore, we have $f_{G}(i, j, t)=1$, if $\left|\left[U_{t}(G)\right]_{i, j}\right|=1$.

\section{ALL-TO-ALL NETWORKS}

In this section, we consider a network in which all sites interact directly. This network is modeled by the complete graph $K_{n}$ on $n$ vertices. In $K_{n}$, we have $d(i)=n-1$, 
for every $i \in V(G)$. The principal result of the section is the following statement:

Theorem 4 Let $K_{n}$ be the complete graph on $n$ vertices. For every vertex $i \in V\left(K_{n}\right)$ and value $n \in \mathbb{N}$, we have the following:

- $\min _{t} f_{K_{n}}(i, i, t)=1-2 / n$ and $t=\frac{\pi}{2 n}+\frac{\pi k}{n}$, for $k \geq 0$;

- $\max _{t} f_{K_{n}}(i, i, t)=1$ and $t=\pi k / n$, for $k \geq 1$.

For every two distinct vertices $i, j \in V\left(K_{n}\right)$ and value $n \in \mathbb{N}$, we have the following:

- $\min _{t} f_{K_{n}}(i, j, t)=0$ is attained when $f_{K_{n}}(i, i, t)$ is maximum;

- $\max _{t} f_{K_{n}}(i, j, t)=2 / n$ is attained when $f_{K_{n}}(i, i, t)$ is minimum.

Proof. Since $d(i)=n-1$, for every $i \in V\left(K_{n}\right)$, we have $A\left(K_{n}\right)=J_{n}-I_{n}$, where $J_{n}$ is the $n \times n$ all-ones matrix. Then, $L\left(K_{n}\right)=(n-1) I_{n}-\left(J_{n}-I_{n}\right)=n I_{n}-J_{n}$. Given $\left|E\left(K_{n}\right)\right|=\left(\begin{array}{l}n \\ 2\end{array}\right)$, it follows that

$$
H\left(K_{n}\right)=\left(\begin{array}{l}
n \\
2
\end{array}\right) I_{n}-2\left(n I_{n}-J_{n}\right)=\frac{n(n-5)}{2} I_{n}+2 J_{n}
$$

The eigenvalues of $H\left(K_{n}\right)$ are then $\lambda_{1}^{[1]}=n(n-1) / 2$ and $\lambda_{2}^{[n-1]}\left(\left[H\left(K_{n}\right)\right]_{i i}-\left[H\left(K_{n}\right)\right]_{i j}\right)=n(n-5) / 2$. Let us denote by $\vec{J}_{n}$ the all-ones vector of dimension $n$. Since $\left[H\left(K_{n}\right), J_{n}\right]=0$, these two matrices share a common set of eigenvectors. The matrix $J_{n}$ is diagonalized by $F_{n}$, the Fourier transform over the group $\mathbb{Z}_{n}:\left[F_{n}\right]_{i j}=e^{\iota 2 \pi i j / n} \equiv$ $\omega^{i j}$. As a consequence, the single eigenvector corresponding to the eigenvalue $\lambda_{1}$ is of the form $\left|\lambda_{1}\right\rangle=n^{-1 / 2} \vec{J}_{n}$; the $n-1$ eigenvectors corresponding to $\lambda_{2}$ are of the form $\left|\lambda_{2}^{i}\right\rangle:=n^{-1 / 2} \sum_{j=1}^{n} \omega^{i j}|j\rangle$, for every $i=1, \ldots, n-1$. Now, we can read $U_{t}\left(K_{n}\right) \equiv e^{-\iota H\left(K_{n}\right) t}$ in its spectral decomposition:

$$
U_{t}\left(K_{n}\right)=n^{-1}\left(e^{-\iota \lambda_{1} t} J_{n}+e^{-\iota \lambda_{2} t} \sum_{i ; j, k=1}^{n-1 ; n} \omega^{i j} \bar{\omega}^{i k}|j\rangle\langle k|\right) .
$$

Then

$$
\left[U_{t}\left(K_{n}\right)\right]_{i i}=n^{-1} e^{-\iota\left(\begin{array}{c}
n \\
2
\end{array}\right) t}+\frac{n-1}{n} e^{-\iota \frac{n(n-5)}{2} t}
$$

for every $i$ and

$$
\left[U_{t}\left(K_{n}\right)\right]_{i j}=n^{-1}\left(e^{-\iota\left(\begin{array}{c}
n \\
2
\end{array}\right) t}-e^{-\iota \frac{n(n-5)}{2} t}\right)
$$

if $i \neq j$. Thus, we have $1-2 / n \leq\left|\left[U_{t}\left(K_{n}\right)\right]_{i i}\right| \leq 1$, for every $i$. The minimum is attained when $t=\frac{\pi \bar{k}}{n}+\frac{\pi}{2 n}$. The maximum is attained for each $t=\pi k / n$. So, $0 \leq$ $\left|\left[U_{t}\left(K_{n}\right)\right]_{i j}\right| \leq 2 / n$. For these entries, the minimum value is attained for each $t$ such that $\left|\left[U_{t}\left(K_{n}\right)\right]_{i i}\right|$ is maximum and viz.

The results is that there is no PST in $K_{n}$ (but for the trivial cases $n \neq 1,2)$. This is equivalent to say that a qubit can not be accurately transferred between two sites in a network in which all sites are connected to each other. The fidelity given by the evolution in $K_{n}$, with $n=4,8$ is illustrated in the figures below (left and right, resp.) for $t \in[0, \pi]$. The dashed lines represent $\left|\left[U_{t}\left(K_{n}\right)\right]_{i j}\right|$ with $i \neq j$; the solid ones, $\left|\left[U_{t}\left(K_{n}\right)\right]_{i i}\right|$.

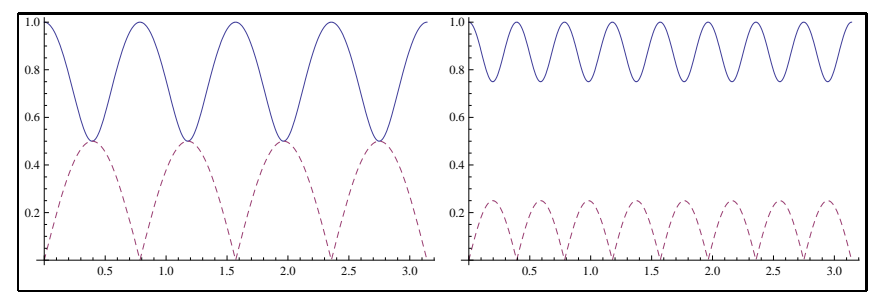

For $t=\frac{\pi k}{n}+\frac{\pi}{2 n}$, Theorem 4 prompts to the following facts. If $n$ is odd then

$$
\left[U_{t}\left(K_{n}\right)\right]_{i j}= \begin{cases}\delta_{i j}-2 \iota / n, & \text { if } n=4 k-1 \\ \delta_{i j}-2 / n, & \text { if } n=4 k+1\end{cases}
$$

If $n$ is even, we need to distinguish two cases:

$$
\begin{aligned}
& \text { - } n=4 k, \\
& {\left[U_{t}\left(K_{n}\right)\right]_{i j}= \begin{cases}-\left(\delta_{i j}-2 / n\right) \sqrt{i}, & \text { if } k \text { even; } \\
\left(\delta_{i j}-2 / n\right) \sqrt{i}, & \text { if } k \text { odd; }\end{cases} } \\
& \text { - } n=4 k+2, \\
& {\left[U_{t}\left(K_{n}\right)\right]_{i j}= \begin{cases}\frac{-\left(\delta_{i j}-2 / n\right) \sqrt{i},}{\left(\delta_{i j}-2 / n\right) \sqrt{i},} & \text { if } k \text { even; } k \text { odd } .\end{cases} }
\end{aligned}
$$

This is the Grover operator with an overall phase. Recall that this operator plays a central role in the Grover algorithm for database search. The unitary $U_{t}\left(K_{n}\right)$ induces a continuous-time quantum walk on $K_{n}$. Notably an alternative version of this search technique has been designed as a discrete-time quantum walk algorithm [8].

\section{ALL-TO-ALL NETWORKS WITH A MISSING LINK}

In this section, we consider a network in which all sites but two interact directly. This network is modeled by $K_{n}^{-}$, the complete graph minus an edge on $n$ vertices. The graph $K_{n}^{-}$is obtained from $K_{n}$ by deleting an arbitrary edge. 
Theorem 5 Let $K_{n}^{-}$be the complete graph minus an edge on $n$ vertices. For every vertex $i \in V\left(K_{n}^{-}\right)$and value $n=4 k$, with $k \in \mathbb{N}$, we have the following statements:

If $i=1, n$ then

- $\min _{t} f_{K_{n}^{-}}(i, i, t)=0$ and $t=\frac{\pi}{4}+\frac{\pi k}{2}$, for $k \geq 0$;

- $\max _{t} f_{K_{n}^{-}}(i, i, t)=1$ and $t=\pi k / 2$, for $k \geq 1$.

If $i \neq 1, n$ then

- $\min _{t} f_{K_{n}^{-}}(i, i, t)=1-2 / n$ and $t=\frac{\pi}{2 n}+\frac{\pi k}{n}$, for $k \geq 0$;

- $\max _{t} f_{K_{n}^{-}}(i, i, t)=1$ and $t=\pi k / n$, for $k \geq 1$.

If $i=1$ and $j=n$ then

- $\min _{t} f_{K_{n}^{-}}(1, n, t)=0$ is attained when $f_{K_{n}}(1,1, t)$ is maximum;

- $\max _{t} f_{K_{n}^{-}}(1, n, t)=1$ is attained when $f_{K_{n}}(1,1, t)$ is minimum.

In all other cases, when $i \neq j($ and $i \neq 1, n)$

- $\min _{t} f_{K_{n}^{-}}(i, j, t)=0$ is attained when $f_{K_{n}}(i, i, t)$ is maximum;

- $\max _{t} f_{K_{n}^{-}}(i, j, t)=2 / n$ is attained when $f_{K_{n}}(i, i, t)$ is minimum.

Proof. Let us define the $n \times n$ matrix $P_{n}$ such that $\left[P_{n}\right]_{1,1}=\left[P_{n}\right]_{n, n}=1,\left[P_{n}\right]_{1, n}=\left[P_{n}\right]_{n, 1}=-1$, and $\left[P_{n}\right]_{i j}=0$, otherwise. The Laplacian of $K_{n}^{-}$can be written as

$$
\begin{aligned}
L\left(K_{n}^{-}\right) & =\left(\begin{array}{ccccc}
n-2 & -1 & \cdots & -1 & 0 \\
-1 & n-1 & -1 & \cdots & -1 \\
\vdots & -1 & \ddots & -1 & \vdots \\
-1 & \cdots & -1 & n-1 & -1 \\
0 & -1 & \cdots & -1 & n-2
\end{array}\right) \\
& =L\left(K_{n}\right)-P_{n} .
\end{aligned}
$$

The XYZ adjacency matrix of $K_{n}^{-}$has then the form

$$
\begin{aligned}
H\left(K_{n}^{-}\right) & =\left(\left(\begin{array}{l}
n \\
2
\end{array}\right)-1\right) I_{n}-2 L\left(K_{n}^{-}\right) \\
& =\left(\left(\begin{array}{l}
n \\
2
\end{array}\right)-1\right) I_{n}-2\left(n I_{n}-J_{n}-P_{n}\right) .
\end{aligned}
$$

This matrix elements are of the form $\left[H\left(K_{n}^{-}\right)\right]_{1, n}=$ $\left[H\left(K_{n}^{-}\right)\right]_{n, 1}=0, \quad\left[H\left(K_{n}^{-}\right)\right]_{i, i}=m-2 d(i)$ and $\left[H\left(K_{n}^{-}\right)\right]_{i, j}=2$. The eigenvalues of $H\left(K_{n}^{-}\right)$are then $\lambda_{1}^{[1]}=n(n-1) / 2-1, \lambda_{2}^{[1]}=\left[H\left(K_{n}^{-}\right)\right]_{1,1}=n(n-$ 1) $/ 2-2 n+3$ and $\lambda_{3}^{[n-2]}=\left[H\left(K_{n}^{-}\right)\right]_{2,2}-\left[H\left(K_{n}^{-}\right)\right]_{1,2}=$ $n(n-5) / 2-1$. We can chose an orthonormal basis of eigenvectors such that, from the spectral decomposition of the unitary matrix $U_{t}\left(K_{n}^{-}\right) \equiv e^{-\iota H\left(K_{n}^{-}\right) t}$, we have the following diagonal entries:

$$
\left[U_{t}\left(K_{n}^{-}\right)\right]_{i i}=\left\{\begin{array}{l}
n^{-1}\left(\frac{n}{2} e^{-\iota\left[\left(\begin{array}{c}
n \\
2
\end{array}\right)-2 n+3\right] t}+e^{-\iota\left[\left(\begin{array}{l}
n \\
2
\end{array}\right)-1\right] t}\right. \\
\left.+\left(\frac{n}{2}-1\right) e^{-\iota\left[\frac{n(n-5)}{2}-1\right] t}\right), \\
\text { if } i=1, n ; \\
n^{-1}\left(e^{-\iota\left[\left(\begin{array}{c}
n \\
2
\end{array}\right)-1\right] t}+(n-1) e^{-\iota\left[\frac{n(n-5)}{2}-1\right] t}\right), \\
\text { otherwise. }
\end{array}\right.
$$

Let us first consider the minimum value of $f_{K_{n}^{-}}(i, i, t)$. In general, we would need to distinguish two cases depending on the parity of $n$. Here we take $n$ to be a multiple of 4 . This implies that $n$ is always even. A simple calculation shows the next facts. It follows that $\min _{t} f_{K_{n}^{-}}(1,1, t)=0$ and $\max _{t} f_{K_{n}^{-}}(1,1, t)=1$ if $t=\frac{\pi}{4}+\frac{\pi k}{2}(k \geq 0)$, and $t=\pi k / 2(k \geq 1)$, respectively. The same holds for $i=n$. If $i \neq 1, n$, it follows that $\min _{t} f_{K_{n}^{-}}(i, i, t)=1-2 / n$ and $\max _{t} f_{K_{n}^{-}}(i, i, t)=1$ if $t=\frac{\pi}{2 n}+\frac{\pi k}{n}$ and $t=\pi k / n$, respectively.

The off-diagonal entries of $U_{t}\left(K_{n}^{-}\right)$are

$$
\left[U_{t}\left(K_{n}^{-}\right)\right]_{i j}=\left\{\begin{array}{l}
n^{-1}\left(e^{-\iota\left[\left(\begin{array}{c}
n \\
2
\end{array}\right)-1\right] t}-\frac{n}{2} e^{-\iota\left[\left(\begin{array}{c}
n \\
2
\end{array}\right)-2 n+3\right] t}\right. \\
\left.+\left(\frac{n}{2}-1\right) e^{-\iota\left[\frac{n(n-5)}{2}-1\right] t}\right) \\
\text { if } i=1 \text { and } j=n \\
n^{-1}\left(e^{-\iota\left[\left(\begin{array}{c}
n \\
2
\end{array}\right)-1\right] t}-e^{-\iota\left[\frac{n(n-5)}{2}-1\right] t}\right), \\
\text { otherwise. }
\end{array}\right.
$$

Given this equation, for $i=1$ and $j=n$, we have $\min _{t} f_{K_{n}^{-}}(1, n, t)=0$ when $t=\pi k / 2$ and $\max _{t} f_{K_{n}^{-}}(1, n, t)=1$ when $t=\frac{\pi}{4}+\frac{\pi k}{2}$, i.e., $f_{K_{n}^{-}}(1, n, t)$ is minimum when $f_{K_{n}^{-}}(1,1, t)$ is maximum and viz. The last case to describe is when $i \neq j$ and $i \neq 1, j \neq n$ : $\min _{t} f_{K_{n}^{-}}(i, j, t)=0$ and $\max _{t} f_{K_{n}^{-}}(i, j, t)=2 / n$ if $t=$ $\pi k / n(k \geq 0)$ and $t=\frac{\pi}{2 n}+\frac{\pi k}{n}(k \geq 1)$, respectively.

The fidelity given by the evolution in $K_{n}^{-}$, with $n=$ $4,8,16,40$ is illustrated in the figures below (top L/R, bottom $\mathrm{L} / \mathrm{R}$, resp.) for $t \in[0, \pi]$. The thick lines represent $f_{K_{n}^{-}}(1,1, t)$; the thin ones, $f_{K_{n}^{-}}(1, n, t)$. The dashed lines represent $f_{K_{n}^{-}}(2,2, t)$; the dotted ones, $f_{K_{n}^{-}}(2,3, t)$.

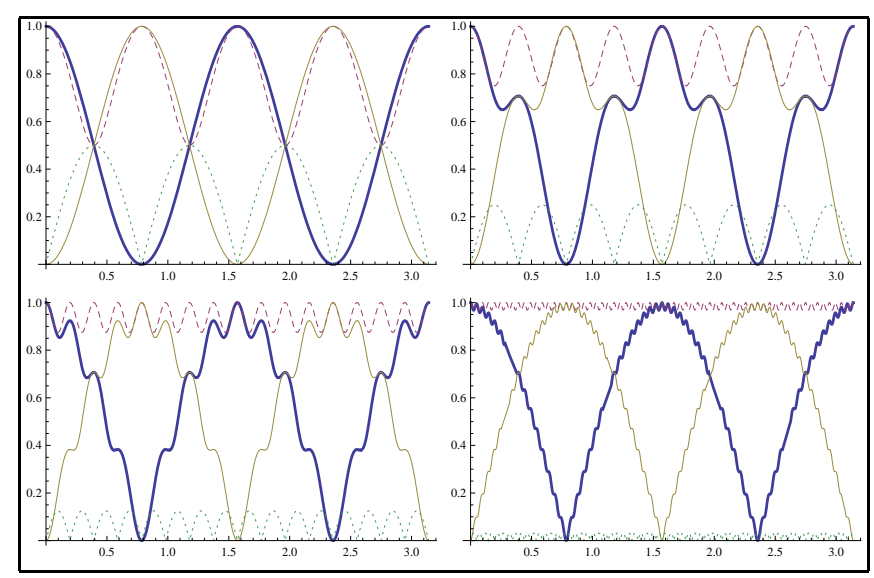



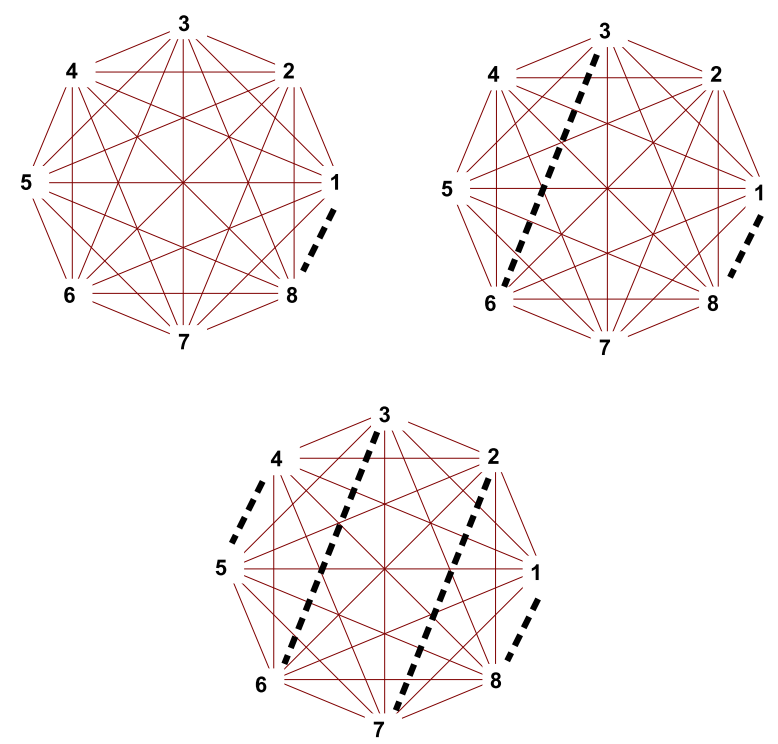

FIG. 1: There is PST between vertex 1 and 8 in the top-left graph; for the pairs $\{1,8\}$ and $\{3,6\}$ in the top-right one; for the pairs $\{1,8\},\{2,7\},\{3,6\},\{4,5\}$ in the bottom graph. The dashed lines represent deleted edges.

When $n$ is a multiple of 4 , the unitary $U_{t}\left(K_{n}^{-}\right)$can be used to route a qubit over $n$ sites. To put in communica- tion site $i$ and site $j$, we need to let the system evolve for a time $t=\frac{\pi}{4}+\frac{\pi k}{2}$, once we have deleted from $K_{n}$ exactly the edge $i j$.

The setting described above can be generalized by deleting more than a single edge from $K_{n}$. In fact, as far as we delete edges without common vertices (or, in other words, vertex disjoint edges), we still can obtain PST between the end points of deleted edges, whenever the number of vertices in the graph is a multiple of 4 . The maximum number of edges that can be deleted is then $n / 2$. In this case, the deleted set corresponds to a perfect matching, that is, a set of vertex disjoint edges including all the vertices of the graph. As it is for $K_{n}^{-}$, PST occurs when $t=\frac{\pi}{4}+\frac{\pi k}{2}$. Figure 1 describes an example.

\section{CONCLUSION}

We have given an explicit relation between the Hamiltonian for networks of interacting qubits in $X Y Z$ model and the graph Laplacian. We have studied evolution in networks modeled by the complete graph $K_{n}$ and the complete graph minus one edge $K_{n}^{-}$. While $K_{n}$ does not allow PST, we have seen that $K_{n}^{-}$does allows PST between the two non-adjacent sites, when $n$ is a multiple of four. This result can be used to arbitrarily route a qubit between any two vertices of $K_{n}^{-}$.
[1] A. Bernasconi, C. Godsil, S. Severini, preprint. arXiv:0808.0510v1 [quant-ph]

[2] S. Bose S, Phys. Rev. Lett. 91207901 (2003). arXiv:quant-ph/0212041 2

[3] S. Bose, Contemporary Physics, Vol. 48 (1), pp. 13-30, 2007. arXiv:0802.1224v1 [cond-mat.other]

[4] D. Burgarth, S. Bose, Phys. Rev. A 71, 052315 (2005). arXiv:quant-ph/0406112 4

[5] M. Christandl, N. Datta, A. Ekert and A. J. Landahl, Phys. Rev. Lett. 92, 187902 (2004). arXiv:quant-ph/0309131 22

[6] K. Eckert, O. Romero-Isart, A. Sanpera, New J. Phys. 9
(2007) 155. arXiv:quant-ph/0702082 2

[7] C. Facer, J. Twamley, J. D. Cresser, Phys. Rev. A 77, 012334 (2008). arXiv:0706.3821 v1 [quant-ph]

[8] F. Magniez, A. Nayak, J. Roland, M. Santha, Search via quantum walk. In: Proc. of the 39th ACM Symposium on Theory of Computing, pp. 575-584 (2007). arXiv:quant-ph/0608026 3

[9] T. J. Osborne, N. Linden, Phys. Rev. A 69052315 (2005). arXiv:quant-ph/0312141 v1

[10] V. Subrahmanyam, Phys. Rev. A 69034304 (2004). arXiv:quant-ph/0307135v2 